\title{
How effective is it to design by voice?
}

\author{
Mohammad M. Alsuraihi \\ Department of Computing \\ University of Bradford \\ Bradford, West Yorkshire, BD7 1DP \\ 00441274236830 \\ mmnalsur@bradford.ac.uk
}

\author{
Dimitris I. Rigas \\ Department of Computing \\ University of Bradford \\ Bradford, West Yorkshire, BD7 1DP \\ 00441274235131 \\ d.rigas@bradford.ac.uk
}

\begin{abstract}
Previous studies on usability of crowded graphical interfaces that are full of widgets like menus, buttons, palette-tools etc, have shown evidence that they create a fertile environment for information overload and usability problems. In this paper, we investigate the use of multimodal interaction metaphors (visual, vocal and aural) for improving effectiveness of learning functions and completing tasks in one of the most graphically crowded user-interfaces, the user-interface of IDEs (or Interface Design Environments). This investigation was done empirically on two experimental interface design toolkits (TVOID and MMID) which were built especially for the study. Assessment of the visual and multimodal interaction metaphors was carried out by two independent groups of users (A and B) of which each consisted of 15 users. Results showed that the use of speech for input and output along with limited use of the mouse was more effective than interacting visually only using the typical common graphical metaphors: pull-down menus, toolbar, toolbox, properties-table and status-bar.
\end{abstract}

\section{Categories and Subject Descriptors}

H.5.2 [Information Interfaces and Presentation]: User Interfaces

\section{General Terms}

Measurement, Performance, Design, Experimentation.

\section{Keywords}

Interface design, Multimodal interaction, Speech recognition, Effectiveness, Usability.

\section{INTRODUCTION}

Crowding the interface with graphical widgets that allow visual interaction only with no involvement of other senses like the auditory system exposes the user to experience information overload [1]. This causes important information around the interface to be missed [2]. Another problem with visual-only interaction is the high potential for usability problems with graphical metaphors to occur. There are two root-problems from which all usability problems branch: interface intrusion into task [3] and closure [4]. The key solution to enhancing usability of graphical interfaces is to lessen the visual workload on the visual channel, which negatively affects effectiveness of

(c) Mohammad M. Alsuraihi \& Dimitris I. Rigas, 2007.

Published by the British Computer Society

Volume 2 Proceedings of the 21 st BCS HCI Group Conference

HCI 2007, Lancaster University, 3-7 September 2007

Devina Ramduny-Ellis \& Dorothy Rachovides (Editors) task-performance [5]. In order to enhance usability of a userinterface, more than one modality has to be involved in interaction. In this paper, we investigate the use of multimodal interaction metaphors (visual and auditory) for improving effectiveness of interface design toolkits. The paper presents a multi-group study comparing effectiveness of speech as input and output means for designing user-interfaces against effectiveness of the common typical GUIs that can be used for the same purpose. The study targeted novel computer users who had limited knowledge in using Interface Design Environments (IDEs). In order to fulfill the aim, two interface design toolkits were built from scratch (TVOID and MMID) to be tested empirically. The following sections present an overview of the related work and put more light on the experimental toolkits, design of the experiments and discussion of the results obtained.

\section{RELEVANT WORK}

Previous researchers recommend the addition of non-speech sounds (earcons $[6,7]$ and auditory icons $[8,9]$ ) to interfaces in order to improve their performance and increase their usability. However, in order for perception and right interpretation of non-speech sound to be successfully achieved, a high level of concentration and the development of a perceptual context are required by the users $[7,10]$. This causes the users to incorrectly interpret the non-speech messages sometimes, because of lack of concentration and distraction with other events or messages in the interface [10]. Processing natural language (speech) feedback in the interface has been recognized as bringing many benefits to human-computer interaction [11]. Rigas et al $[10,12]$ investigated the use of speech along with non-speech sounds, and found that combining earcons with synthesized speech was a successful and effective approach for communicating information to the users. In addition, a study by Vargas and Anderson showed that the users' performance was better in terms of time, number of keystrokes, errors, and workload when used speech along with earcons [13].

The work by Bolt [14], which introduced the approach of processing speech and gesture for moving graphical objects on an interface, was pioneer and promising for researchers to investigate the use of speech as an input utility for enhancing efficiency of the user-interface. Cohen, Oviatt and others [1519] have strongly recommended employment of speech recognition for utilizing the user interface. Recent studies have shown the potential of this interaction metaphor for enhancing performance of interaction between the user and the interface [20-24]. However, all these studies have discussed effectiveness of speech recognition with caution due to recognition errors that emerge from sensitivity toward noise and inaccurate pronunciation of words. These errors are tamable and can be tolerated $[17,25]$, especially if limited vocabulary was used [26]. Also, the impact of such errors can be dramatically 
reduced if the speech-recognition system being studied was compared to a counterpart visual-only graphical system in regard to usability measures. Our review of the previous related work has revealed a strong need for such comparative usability studies. In this study we measure effectiveness of vocal instruction against effectiveness of visual-only instruction for designing interfaces using two experimental Interface Design toolkits (TVOID and MMID).

\section{EXPERIMENTAL TOOLKITS}

Two experimental toolkits were developed using Microsoft Visual C\#: Typical Visual-Only Interface Design (TVOID) and Multi-Modal Interface Design (MMID) toolkits. TVOID imitates the style of interaction implemented in most of the existing interface-design environments like Microsoft Visual C\# and Java NetBeans IDE. It interacts with the user visuallyonly with no involvement of other senses like the auditory system. This interaction takes place in six areas in its main interface: menus, toolbar, toolbox, workplace, properties-table, and status-bar. Figure 1 shows a screenshot of TVOID.

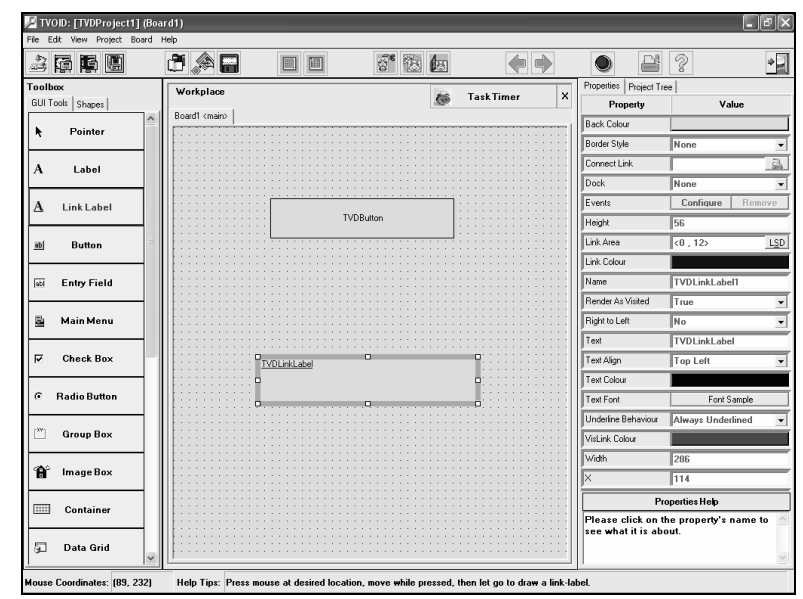

Figure 1: A screenshot of TVOID's main interface

MMID provides a combination of visual, vocal and aural interaction metaphors. It is a speech-recognition and text-tospeech based environment that allows limited use of the mouse and the keyboard. It allows the user to interact with it from the position of the mouse-cursor. In this environment, there is no need for the user to use any of the graphical metaphors implemented in TVOID. The system command receptor in this environment is represented by a friendly character (MS Agent) that listens to commands and interacts with the user via speech and facial expressions. Vocal commands are in the form of simple one to three English-words. Figure 2 shows a screenshot of MMID.

\section{DESIGN OF THE EMPIRICAL STUDY}

The empirical study aimed at measuring effectiveness of learnability (or the ability to learn functions from first time use) of TVOID and MMID. The toolkits were tested independently by two groups of users (A and B). Each group consisted of 15 users. The participants were computer users who had limited experience in using interface design environments. Each user attended a 10-minute training video for using the toolkit he/she was assigned to test. Both groups were asked to complete the same tasks (10 tasks). Each task consisted of one to three functions. The tasks were designed to be increasing in complexity and covering all expected functionality (activating menu-command functionality, selecting tools, drawing objects, and setting properties). Effectiveness was measured by calculating the percentage of functions learned in absence of additional help and percentage of tasks completed successfully (i.e. within task-completion criterion time). In order to obtain these percentages, efficiency of the two experimental toolkits was first measured by timing learning functions and completing tasks for each user under the two conditions (visual-only and multimodal).

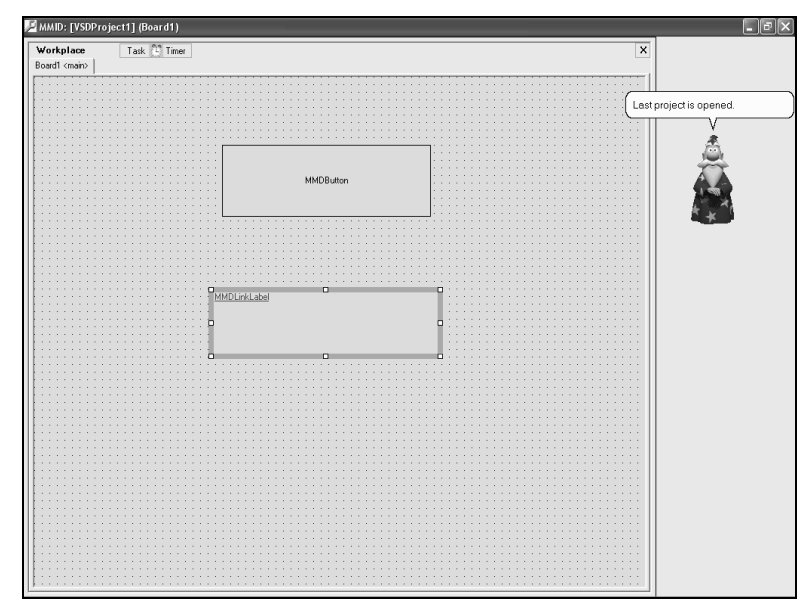

Figure 2: A screenshot of MMID's main interface

\section{DISCUSSION OF RESULTS}

During the experiments, it was noticed that the users who tested TVOID (Group A) expected how to do most of the functions. This environment looked familiar to them because they had previous experience with similar environments that provided with similar interaction metaphors. This experience made them primarily rely on their memory. Before doing a task, the users of TVOID spent time on recalling how to do functions in the similar systems they were used to, to be able to do them using this environment. Expectations of how to do functions were incorrect sometimes, which caused the users to find out how to do these functions. In this way, the users of TVOID did two things to learn functions: remembering or expecting, and exploring in case of incorrect expectation. This was not the case with the users who tested MMID (Groups B) as they were not familiar to voice-instruction, and thus they headed directly to exploring. Although MMID was more prone to errors than TVOID because of sensitivity toward noise and accurate pronunciation of words, it must be recalled that it was tried for the first time and that frequent use could lessen the number of errors and make it more usable.

Figure 3 shows the variances between the two environments in regard to task accomplishment (learning and completion) time. These variances have mainly taken place because of the methods used to learn functions. Another factor behind this result was that MMID limited the use of the mouse and decreased the user-reliance on the visual sense. Significance of the difference was tested using the t-test. The difference was found significant $(\mathrm{t}=2.64, \mathrm{P}=0.02)$. Gathering all commands in one location (e.g. one list) in MMID helped the users in Group B to locate the required commands for doing functions in a faster way than their counterparts in Group A, who looked for these commands in different locations around the interface. Also, the use of one interaction metaphor (voice-instruction) in MMID with limited use of the mouse and the keyboard saved the time for the users to think where to go to activate the required command within the many encapsulated widgets in the 
interface (menus, toolbar, drawing tools, properties, etc) as in TVOID. For example, In order to draw an object in TVOID, the user must select it at first from the tool-box, while in MMID the user can draw directly by saying the tool's name on the required location. Also, learning how to set properties was done through interactive training simulations in MMID, while in TVOID was done textually. Enabling the user to specifically learn what he/she needs to learn using interactive training as in MMID saves the time for thinking of the appropriate keywords, looking for them, and reading about them as in TVOID.

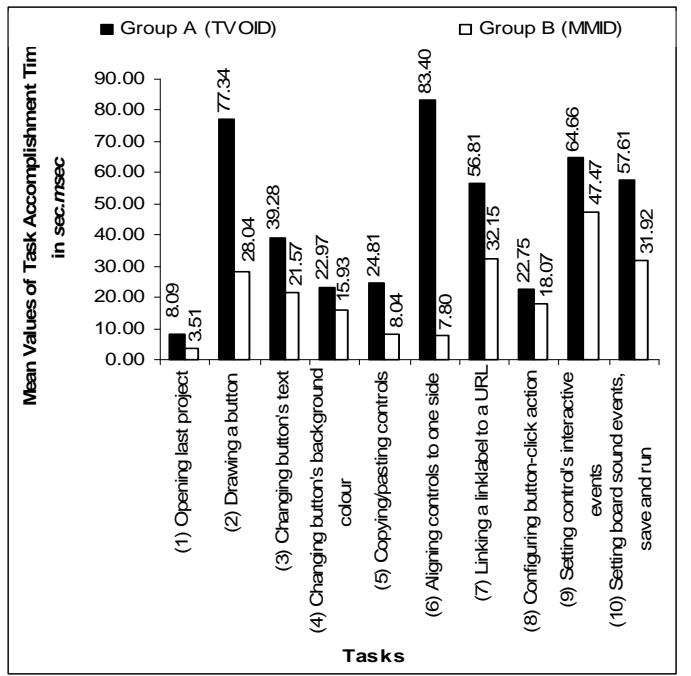

Figure 3: Mean values of time taken for accomplishing 10 tasks for the first time using TVOID (Group A) and MMID (Group B)

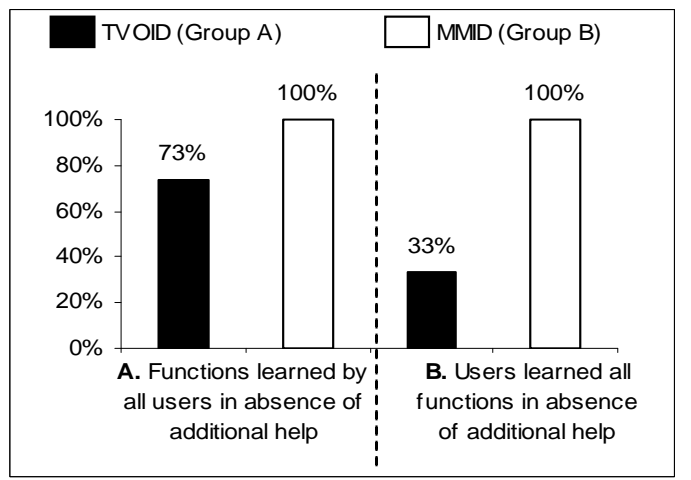

Figure 4: Percentages for the functions learned by all users and users who learned all functions in absence of additional help using TVOID and MMID

An efficient system that helps the user to learn functions within the least possible time is considered to be effectively learnable because the probability that all required functions would be learned by all users is high. Similarly, the probability that all users would learn all functions if the system is pretty efficient is also high. Figure 4.A and Figure 4.B reinforce this finding. This also applies for effectiveness of task completion in terms of percentages of tasks completed successfully (i.e. within threshold time) by all users and the percentage of users who complete all tasks successfully as can be seen in Figure 5.

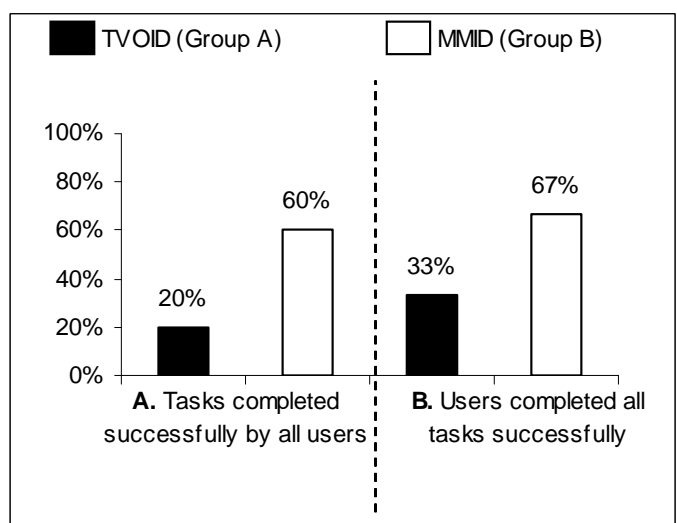

Figure 5: Percentages for (A) the tasks completed successfully by all users and (B) the users who successfully completed all tasks using TVOID and MMID

\section{Conclusion}

In this paper, we provided with a comparative study done by two independent groups of novel users who had limited knowledge in using IDEs. The study aimed at measuring effectiveness of two interface design toolkits: typical graphical and visual-only toolkit (TVOID), and a speech input and output toolkit (MMID). TVOID interacted with the user through six areas comprising pull-down menus, toolbar-buttons, drawing tools, workplace (form being designed), properties table and status-bar. On the other hand, MMID interacted with the user through one area only, which is the workplace. All the other graphical interaction metaphors were replaced by vocal commands. From results, it can be concluded that in order to shorten task accomplishment time and, hence, enhance effectiveness of task accomplishment, a design environment should aim at enabling the user to do all actions from inside the workplace area with no need to leave it to other areas. The more visual interaction metaphors an environment provides the more time will be spent in thinking where to find the appropriate ones for accomplishing jobs, and the vice-versa is correct. The use of voice-instruction as a way of interaction was found to be more effective than the use of several visual interaction metaphors, in terms of shortening function learning and taskaccomplishment time. This study opens a research door toward substituting the well-known graphical interaction metaphors used in IDEs, namely, menus, toolbar, toolbox, properties-table and status-bar with vocal commands in order to enhance usability of the user-interface of ID systems.

\section{Future work}

The empirical work covered in this paper investigated the usability parameters: efficiency and effectiveness for the two experimental environments from one angle: learnability or the ability to accomplish tasks from first time use. Further experiments will take place for measuring Experienced User Performance (EUP) using the same experimental toolkits. More than 30 users will be recruited for each environment to make the samples more representatives of the target population. In addition, user-satisfaction with the interaction metaphors provided in each environment will be empirically evaluated.

\section{ACKNOWLEDGMENTS}

Our thanks to ACM SIGCHI for allowing us to modify templates they had developed. 


\section{REFERENCES}

[1] Brewster, S. A., "Using Non-Speech Sound to Overcome Information Overload," Displays, vol. 17, pp. 179-189, 1997.

[2] Oakley, I., McGee, M. R., Brewster, S., and Gray, P. D., "Putting the feel in look and feel," In ACM CHI 2000 (The Hague, NL), pp. 415-422, 2000.

[3] Brewster, S. A. and Clarke, C. V., "The Design and Evaluation of a Sonically Enhanced Tool Palette," ACM Transactions on Applied Perception (TAP), vol. 2, pp. 455-461, 2005

[4] Dix, A., Finlay, J., Abowd, G., and Beal, R., HumanComputer Interaction, Second ed: Prentice Hall, 1998.

[5] Brown, M. L., Newsome, S. L., and Glinert, E. P., "An Experiment into the Use of Auditory Cues to Reduce Visual Workload," In proceedings of ACM CHI '89, Austin, Texas, USA, pp. 339-346, 1989.

[6] Rigas, D. I. and Atly, J. L., "The Rising Pitch Metaphor: An Empirical Study," International Journal of HumanComputer Studies, vol. 62, pp. 1-20, 2005.

[7] Rigas, D., Memery, D., Hopwood, D., and Rodrigues, M., "Using Non-Speech Sound to Communicate Information in User Interfaces," In Applied Informatics 2000, Innsbruck, Austria, pp. 357-362, 2000.

[8] Gaver, W., "Auditory Interfaces," in Handbook of Human Computer Interaction, vol. 1, M. G. Helander, T. K. Landauer, and P. V. Prabhu, Eds. Amsterdam: Elsevier, 1997, pp. 1003-1041.

[9] Cohen, M., "Throwing, Pitching and Catching Sound: Audio Windowing Models and Modes," International Journal of Man-Machine Studies, vol. 39, pp. 269-304, 1993.

[10] Rigas, D. I. and Memery, D., "Utilising Audio-Visual Stimuli in Interactive Information Systems: A Two Domain Investigation on Auditory Metaphors," In International Conference on Information Technology: Coding and Computing, pp. 190-195, 2002.

[11] Manaris, B., "Natural Language Processing: A HumanComputer Interaction Perspective," Advances in Computers, vol. 47, pp. 1-66, 1996.

[12] Rigas, D., Yu, H., and Memery, D., "Experiments Using Speech, Non-Speech Sound and Stereophony as Communication Metaphors in Information Systems," In proceeding of the 27th Euromicro Conference, Warsaw, Poland, pp. 383-390, 2001.

[13] Vargas, M. L. and Anderson, S., "Combining Speech and Earcons to Assist Menu Navigation," pp., 2003.

[14] Bolt, R. A., "Put-that-there: Voice and Gesture at the Graphics Interface," In proceedings of the 7th annual conference on Computer Graphics and Interactive Techniques Seattle, Washington, USA, pp. 262-270, 1980.
[15] Cohen, M. and Ludwig, L. F., "Multidimensional Audio Window Management," International Journal of ManMachine Studies, vol. 34, pp. 319-336, 1991.

[16] Cohen, P. R., Johnston, M., McGee, D., Oviatt, S. L., Clow, J., and Smith, I., "The Efficiency of Multimodal Interaction: A Case Study," In proceedings of the International Conference on Spoken LAnguage, Sydney, Australia, pp., 1998.

[17] Cohen, P. R. and Oviatt, S. L., "The Role of Voice Input for Human-Machine Communication," In proceedings of the National Academy of Sciences, pp. 9921-9927, 1995.

[18] Mellor, B. A., Baber, C., and Tunley, C., "Evaluating Automatic Speech Recognition as a Component of a Multi-Input Human-Computer Interface," In proceedings of the International Conference on Spoken Language, 1996.

[19] Cohen, P. R., Johnston, M., McGee, D., Oviatt, S. L., Pittman, J., Smith, I., Chen, L., and Clow, J., "QuickSet: Multimodal Interaction for Distributed Applications," In proceedings of the Fifth ACM International Multimedia Conference, New York, pp. 31-40, 1997.

[20] ElAarag, H. and Schindler, L., "A Speech Recognition and Synthesis Tool," In proceedings of the 44th Annual Southeast Regional Conference, Melbourne, Florida, pp. 45-49, 2006.

[21] Adler, A. and Davis, R., "Speech and Sketching for Multimodal Design," In International Conference on Computer Graphics and Interactive Techniques, Boston, Massachusetts, pp. 214-216, 2006.

[22] Jung, J. H., Looney, C. A., and Valacich, J. S., "FineTuning the Human-Computer Interface: Verbal versus Keyboard Input in an Idea Generation Context," In proceedings of the 40th Hawaii International Conference on System Sciences (HICSS'07), pp. 27c, 2007.

[23] Begel, A. and Graham, S. L., "An Assessment of a SpeechBased Programming Environment," In Visual LAnguages and Human-Centric Computing (VL/HCC'06), pp. 116120, 2006.

[24] Desilets, A., Fox, D. C., and Norton, S., "Voce Code: An Innovative Speech Interface for Programming-by-Voice," In Extended Abstracts of the 2006 Conference on Human Factors in Computing Systems (CHI 2006), Montereal, Quebec, Canada, pp., 2006.

[25] Oviatt, S. L., "Taming Recognition with Multimodal Interface," Communications of the ACM, vol. 43, pp. 4551,2000

[26] Lahtinen, S. and Peltonen, J., "Enhancing Usability of UML CASE-Tools with Speech Recognition," In proceedings of IEEE Symposium on Human Centric Computing Languages and Environments, pp. 227-235, 2003. 